\title{
Evaluation of Risk of Emergency Network Public Opinion Based on Method of Fuzzy Grey Evaluation
}

\author{
Pengcheng $\mathrm{Lu}^{1,2, \mathrm{a}^{*}}$ and Naiping Zhang ${ }^{1,2, \mathrm{~b}}$ \\ ${ }^{1}$ Management School, Wuhan University of Technology, Wuhan 430070, China \\ ${ }^{2}$ China Research Center for Emergency Management, Wuhan University of Technology, Wuhan \\ 430070, China \\ a1942634130@qq.com, b541365020@qq.com
}

\section{Keywords: Network public opinion; Emergency; Fuzzy gray evaluation; Life cycle; Risk}

\begin{abstract}
If the response to the emergency public opinion network is not well, the network public opinion crisis will lead to serious damage to public. Therefore, it is urgent to study the risk of emergency network public opinion. This paper, based on life cycle of public opinion, explaines the meaning of emergencies network public opinion and risk structure, and uses fuzzy gray evaluation method to build network public opinion risk indicator evaluation system. It works well to scientifically evaluate and analyze the risk of emergency network public. This method helps the government and enterprises to enhance capacity of response to risks of emergency network public opinion.
\end{abstract}

\section{Introduction}

The In recent years, Chinese emergencies happen frequently, such as "Star of the East Star shipwreck incident", "Tianjin Port bombing", "Shanghai Bund stampede". And the occurrence of emergencies are often accompanied by the outbreak of public opinion network. China has a lot of Internet users and websites. CNNIC (China Internet Network Information Center) released the 38th "China Internet Development Statistics Report" shows that in June 2016, the scale of Chinese Internet users reached 710 million, the Internet penetration rate reached 51.7\% [1]. Network public opinion of the emergency spread fast, and affect many aspects. Emergency network public opinion If not effectively deal with network public opinion, it will cause the crisis of network public opinion, and serious damage to public order. Therefore, it is urgent to study the risk of emergency Network public opinion. Based on the life cycle of network public opinion, this paper discusses the connotation and constitution of public opinion risk in emergent network, and constructs the comprehensive indicator evaluation system by fuzzy gray evaluation method, and analyzes it.

\section{Risks and Components of Emergency Network Public Opinion}

Scientific Connotation. The emergency network public opinion refers to the collection of the public's guided opinions, emotions and attitudes on the network, when an incident occurs [2]. Network public opinion often affect the handling of the incident, and even evolved into another crisis. Therefore, it is necessary to study the risk of the emergency network public opinion in a multi-perspective, comprehensive and continuous way. To prevent emergency network public opinion evolves to the negative and crisis.

Components. Researchers tend to study the network public opinion based on perspective of crisis cycle of network public opinion [3,4]. According to the public opinion life cycle of emergencies, the public opinion risk can be evaluated from the risk of public opinion prevention and control, public opinion formation risk, public opinion spread risk and public opinion recovery risk. The relevant elements of Emergency network public opinion are summarized into three dimensions, namely the object dimension, content dimension and time dimension [5]. The establishment of the evaluation indicator system should follow the principles of systematization, operability and representativeness. This paper analyzes the public opinion of emergent events from 
object dimension, time dimension and content dimension. Time dimension is the network public opinion life cycle. Object dimension in the public opinion audience is the majority of Internet users, and all kinds of information associated with emergencies can be called public opinion information. Public opinion publishers, including Internet users themselves, involved in the generation or dissemination of public opinion information. Public opinion leaders are government and media could guide the public opinion. In the content dimension each object generates, utilizes and disseminates relevant information about the incident through the network. Based on the analysis of public opinion risk in emergent network, the authors consulted with experts on emergency management and website construction. After careful analysis and screening, the corresponding evaluation indicator system was established. Including a total of four first-level indicators and 16 second-level indicators [6,7]. The specific content is as Table 1.

\section{Design of Risk Evaluation Indicator of Emergency Public Opinion}

Determine the Weight of the Evaluation Indicator. Emergency network public opinion risk evaluation indicator system, as shown in Table 1. Let $\boldsymbol{W}$ denote the target layer, which is comprehensive risk of emergency network public opinion. Let $\boldsymbol{U}$ denote the criterion layer. First grade evaluation indicator (prevention and control risk, public opinion formation risk, public opinion spread risk, public opinion recovery risk) is marked as $\boldsymbol{U}_{\boldsymbol{i}}$, then get the set $\boldsymbol{U}=\left\{\boldsymbol{U}_{1}, \boldsymbol{U}_{2}, \boldsymbol{U}_{3}, \boldsymbol{U}_{4}\right\}$. And $\boldsymbol{U}_{\boldsymbol{i}}$ represents the set of the second evaluation indicator $\boldsymbol{U}_{\boldsymbol{i} \boldsymbol{j}}$. We can get the set $\boldsymbol{U}_{\boldsymbol{i}}=\left\{\boldsymbol{U}_{\boldsymbol{i 1}}, \boldsymbol{U}_{\boldsymbol{i} \mathbf{2}}, \boldsymbol{U}_{\boldsymbol{i} \mathbf{3}}, \boldsymbol{U}_{\boldsymbol{i 4}}\right\}$. In this paper, AHP (Analytic Hierarchy Process) is used to construct a hierarchical model of evaluation indicator system for emergencies public opinion risk [8]. Specific results are shown in Table 1.

Table 1 Indicators and weight of assessment of emergency public opinion risk

\begin{tabular}{|c|c|c|c|}
\hline $\begin{array}{l}\text { First level } \\
\text { indicator }\end{array}$ & weight & secondary level indicator & weight \\
\hline \multirow{4}{*}{$\begin{array}{l}\text { Prevention and } \\
\text { control risk } U_{1}\end{array}$} & \multirow{4}{*}{0.2885} & The health of the network environment $U_{11}$ & 0.4637 \\
\hline & & $\begin{array}{l}\text { The Perfection of Public Opinion Monitoring } \\
\text { Platform } U_{12}\end{array}$ & 0.1785 \\
\hline & & Number of Public Opinion Surveillance $U_{13}$ & 0.1661 \\
\hline & & Information disclosure $U_{14}$ & 0.1918 \\
\hline \multirow{4}{*}{$\begin{array}{l}\text { Formation risk } \\
\qquad \mathrm{U}_{2}\end{array}$} & \multirow{4}{*}{0.1819} & Number of related internet users $U_{21}$ & 0.2779 \\
\hline & & Number of Concerned sites $U_{22}$ & 0.1536 \\
\hline & & Number of Concerned sites $U_{23}$ & 0.2228 \\
\hline & & The severity of the event $U_{24}$ & 0.3456 \\
\hline \multirow{4}{*}{ Spread risk $U_{3}$} & \multirow{4}{*}{0.2234} & Page views $\mathrm{U}_{31}$ & 0.1742 \\
\hline & & Degree of interaction $U_{32}$ & 0.2496 \\
\hline & & Emotional tendencies of internet users $U_{33}$ & 0.3483 \\
\hline & & Duration of public opinion $U_{34}$ & 0.2279 \\
\hline \multirow{4}{*}{$\begin{array}{l}\text { Recovery risk } \\
\qquad \mathrm{U}_{4}\end{array}$} & \multirow{4}{*}{0.3062} & Response speed of public opinion $U_{41}$ & 0.1271 \\
\hline & & Event handling satisfaction $\mathrm{U}_{42}$ & 0.2390 \\
\hline & & Guiding ability of public opinion $\mathrm{U}_{43}$ & 0.2935 \\
\hline & & Heat of public opinion $U_{44}$ & 0.2056 \\
\hline
\end{tabular}


Consistency test at all levels, First level indicator, CR $=0.0050<0.10$. Similarly, the CR of the secondary indicator under each indicator is calculated as $0.0238,0.0308,0.0115$ and 0.0038 . We can think through the consistency test.

Determine the set of Reviews and the Sample Matrix. The classification of emergency network public opinion is divided into 5 levels: "very high", "high", "medium", "low"," very low". Then set the comment set toV $=\left\{\mathrm{V}_{1}, \mathrm{~V}_{2}, \mathrm{~V}_{3}, \mathrm{~V}_{4}, \mathrm{~V}_{5}\right\}$. The corresponding scores are 5, 4,3,2,1. When the indicator level is between two adjacent levels, the corresponding score is 4.5, 3.5, 2.5, 1.5 [9].

Invited 10 experts in the field of emergency management and network public opinion constitute an expert evaluation team. For the "Oriental Star shipwreck incident", "Tianjin Port bombing", "Shanghai Bund stampede" three representative of the major emergencies in China, let experts evaluate the indicators. The evaluation matrix D is obtained as follow:

$\boldsymbol{D}=\left[\begin{array}{cccccccccc}3.5 & 4.5 & 3.5 & 3.5 & 4 & 3.5 & 4 & 3.5 & 3.5 & 3 \\ 3 & 3 & 3.5 & 3 & 3.5 & 3 & 3 & 2.5 & 3 & 3 \\ 2.5 & 3 & 3 & 3.5 & 2.5 & 2.5 & 2 & 2.5 & 3 & 3 \\ 3 & 3 & 3 & 3 & 3 & 3.5 & 2 & 2 & 2.5 & 2.5 \\ 4.5 & 4 & 3.5 & 4 & 4.5 & 3.5 & 3.5 & 3 & 3.5 & 4 \\ 4 & 3 & 3 & 3.5 & 3.5 & 3 & 3.5 & 3 & 3.5 & 3.5 \\ 4 & 3.5 & 2.5 & 3 & 3 & 3.5 & 4 & 3.5 & 3.5 & 4 \\ 4.5 & 4.5 & 4 & 4 & 4.5 & 4 & 4 & 4.5 & 5 & 5 \\ 3.5 & 3 & 3 & 3.5 & 3 & 3 & 3.5 & 3.5 & 3.5 & 3.5 \\ 4 & 3.5 & 4 & 3.5 & 3.5 & 3.5 & 3 & 4 & 4 & 4.5 \\ 5 & 4.5 & 4 & 4 & 4 & 4 & 3.5 & 5 & 4 & 4 \\ 3 & 2.5 & 2 & 3 & 3.5 & 3 & 3 & 3 & 3.5 & 3.5 \\ 3 & 3 & 2.5 & 2 & 2.5 & 2 & 3 & 3 & 2 & 3 \\ 2.5 & 3.5 & 3.5 & 3 & 2.5 & 3 & 4 & 4 & 3.5 & 3 \\ 2.5 & 3 & 3 & 2.5 & 3 & 2.5 & 2.5 & 2.5 & 3 & 3 \\ 2 & 2.5 & 3 & 2.5 & 2 & 2 & 2 & 1.5 & 3 & 3\end{array}\right]$

Establish of Evaluation Gray Class and Whiten Weight Function. In this paper, the gray matrix method is used to calculate the weight matrix of the evaluation index [10]. According to the evaluation criterion of the above evaluation indicator $C_{i j}$, five evaluation gray classes are set, and $e=1,2,3,4,5$ is gray classification number, which represent "very high", "high", "medium", "low", "very lo w". Whitening weight function of five evaluation gray classes are shown as follows.

First gray class: "very high" $\{e=1\}$, gray number $\bigotimes_{1}[0,5,10]$, whiten weight function $f_{1}$ :

$$
f_{1}\left(d_{i j k}^{(s)}\right)=\left\{\begin{array}{cc}
(s) & d_{i j k}^{(s)} \in[0,5] \\
d_{i j k} / 5 & d_{i j k}^{(s)} \in[5,10] \\
1 & d_{i j k}^{(s)} \notin[0,10]
\end{array}\right.
$$

Second gray class: "high" $\{e=2\}$, gray number $\bigotimes_{2}[0,4,8]$, whiten weight function $f_{2}$ :

$$
f_{2}\left(d_{i j k}^{(s)}\right)=\left\{\begin{array}{cl}
d_{i j k}^{(s)} / 4 & d_{i j k}^{(s)} \in[0,4] \\
\left(8-d_{i j k}^{(s)}\right) / 4 & d_{i j k}^{(s)} \in[4,8] \\
0 & d_{i j k}^{(s)} \notin[0,8]
\end{array}\right.
$$

Third gray class: "medium" $\{e=3\}$, gray number $\otimes_{3}[0,3,6]$, whiten weight function $f_{3}$ :

$$
f_{3}\left(d_{i j k}^{(s)}\right)=\left\{\begin{array}{cl}
d_{i j k}^{(s)} / 3 & d_{i j k}^{(s)} \in[0,3] \\
\left(6-d_{i j k}^{(s)}\right) / 3 & d_{i j k}^{(s)} \in[3,6] \\
0 & d_{i j k}^{(s)} \notin[0,6]
\end{array}\right.
$$

Fourth gray class: "low" $\{e=4\}$, gray number $\otimes_{4}[0,2,4]$, whiten weight function $f_{4}$ : 


$$
f_{4}\left(d_{i j k}^{(s)}\right)=\left\{\begin{array}{cl}
d_{i j k}^{(s)} / 2 & d_{i j k}^{(s)} \in[0,2] \\
\left(4-d_{i j k}^{(s)}\right) / 2 & d_{i j k}^{(s)} \in[2,4] \\
0 & d_{i j k}^{(s)} \notin[0,4]
\end{array}\right.
$$

Fifth gray class: "very low" $\{e=5\}$, gray number $\bigotimes_{5}[0,1,2]$, whiten weight function $f_{5}$ :

$$
f_{5}\left(d_{i j k}^{(s)}\right)=\left\{\begin{array}{cr}
1 & d_{i j k}^{(s)} \in[0,1] \\
\left(2-d_{i j k}^{(s)}\right) / 1 & d_{i j k}^{(s)} \in[1,2] \\
0 & d_{i j k}^{(s)} \notin[0,2]
\end{array}\right.
$$

Calculation of the gray evaluation coefficient. Calculate the evaluation indicators $U_{i j}$. The gray evaluation coefficient of the $S$ indicator corresponding to the $e$ evaluation gray class is expressed as $x_{i j e}^{(s)}$. It has the following representation:

$$
x_{i j t}^{(s)}=\sum_{k=1}^{p} f_{e}\left(d_{i j e}^{(s)}\right)
$$

The gray evaluation coefficient of each evaluation gray is $x_{i j}^{(s)}$. There are:

$$
x_{i j}^{(s)}=\sum_{t=1}^{5} x_{i j t}^{(s)}
$$

According to the Eq. 1 to the Eq. 7 to calculate the gray class statistics, as follows:

When $e=1$, then $\chi_{111}=7.3000$; When $e=2$, then $\chi_{112}=8.08750$; When $e=3$, then $\chi_{113}=7.8333$; When $e=4$, then $\chi_{14}=2.0000$; When $e=5$, then $\chi_{115}=0$. Therefore, $U_{11}$, the total evaluation coefficient, $\chi_{11}=26.0083$. Similarly, you can calculate the other indicators of gray statistics.

Calculation of Gray Evaluation Weight Vector and Weight Matrix. The $U_{i j}$ gray statistic index should be normalized and the evaluation weight vector can be obtained. According to the evaluation weight vector, we can construct the gray fuzzy evaluation matrix of $U=\left\{U_{1}, U_{2}, U_{3}, U_{4}\right\}$. Marked as $R_{1}, R_{2}, R_{3}, R_{4}$ respectively.

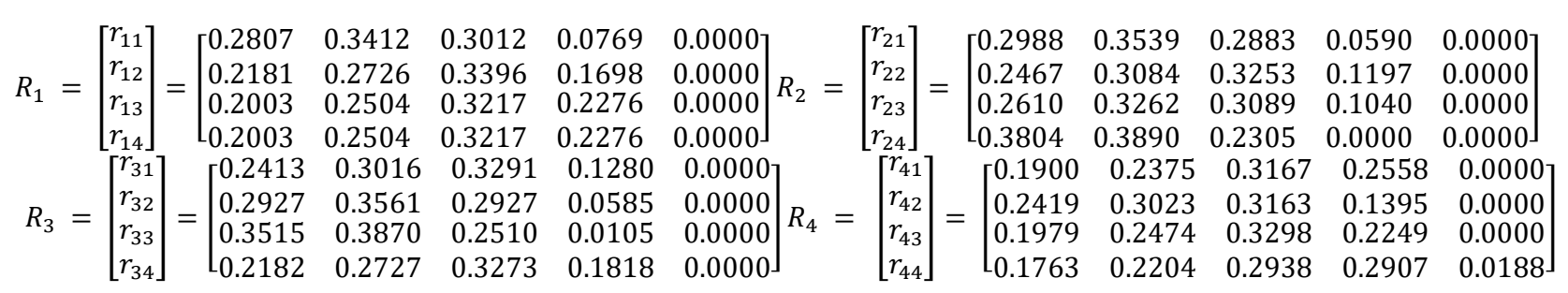

Calculation of the Comprehensive Evaluation Value. Evaluate of the first evaluation indicator $U_{i}$. The evaluation result $B_{i}$ of the first evaluation index $U_{i}$ can be calculated from $B_{i}=\mathrm{W}_{i} * R_{i}$. Then we can get

$$
R=\left[\begin{array}{l}
r 1 \\
r 2 \\
r 3 \\
r 4
\end{array}\right]=\left[\begin{array}{lllll}
0.2407 & 0.2965 & 0.3154 & 0.1474 & 0.0000 \\
0.3106 & 0.3529 & 0.2786 & 0.0580 & 0.0000 \\
0.2872 & 0.3384 & 0.2924 & 0.0820 & 0.0000 \\
0.1763 & 0.2204 & 0.2731 & 0.1916 & 0.0000
\end{array}\right]
$$

The fuzzy comprehensive evaluation value of public opinion risk. From the above calculation, the comprehensive value can be calculated. $\mathrm{B}=(1.2204,1.1711,0.8718,0.2601,0.0012) . e=1,2,3,4,5$ Will be assigned to 5 points, 4 points, 3 points, 2 points, 1 point. The higher the risk, the greater the score. The evaluation gray value grade vector $C=(5,4,3,2,1)$ is used to evaluate the fuzzy comprehensive evaluation value of the public opinion risk of the emergent network: $U=B * C^{T}=3.5247$ 


\section{Analysis of Evaluation Results}

After analyzing the "Star Warship", "Tianjin Port Explosion" and "Stamped on the Bund" with method of the fuzzy comprehensive evaluation, the fuzzy gray comprehensive evaluation value of risk emergency network public opinion is 3.5247. In accordance with the previous evaluation criteria, the Chinese risk of emergency network public opinion is relatively high. So the ability to deal with emergencies network public opinion remains to be improved.

As shown in Table 1, in the first-level indicators, the weight of prevention and control risk and public opinion recovery risk indicators are relatively high $(28.85 \%$ and $30.62 \%)$, but the weight of $\mathrm{A}$ and $\mathrm{B}$ is relatively low $(18.19 \%, 22.34 \%$ respectively). In the secondary indicators, the weight is recovery high as follows: The health of the network environment $(46.37 \%)$, Number of related internet users (27.79\%), Emotional tendencies of internet users (34.83\%), Guiding ability of public opinion (29.35\%). According to the principle of maximum subordination, in terms of the first-level indicators, formation risk and spread risk index evaluation right is relatively high $(0.3529,0.3384)$. In terms of secondary indicators, the weight is recovery high as follows: the health of the network environment (0.3412), the severity of the event (0.3890), emotional tendencies $(0.3870)$, and guiding ability (0.3298). Indicating that the risk is relatively high. The monitoring of these indicators should be strengthened.

\section{Conclusion}

This paper builds up the evaluation model based on public opinion life cycle theory, and uses fuzzy gray comprehensive evaluation method to analyze and evaluate the risk of emergency network public opinion, and finally puts forward some suggestions. The evaluation results show that the risk of China's emergency network public opinion is relatively high. We should pay attention to prevent the spread of public opinion risks, and create a healthy network environment, increase the degree of information disclosure, enhance the user's event handling satisfaction, attention to public opinion-oriented capacity-building. In this paper, fuzzy comprehensive evaluation method is used to evaluate the risk of emergency network public opinion, which is feasible and versatile. Because of the fuzziness and grayness of experts' judgment information, the results are more scientific and reliable.

\section{References}

[1] Information http://www.cnnic.net.cn/hlwfzyj/hlwxzbg/hlwtjbg/201608/P020160803367337470363.pdf (In Chinese)

[2] D.H. Liu, W. G Wang and H.Y Li: Evolutionary Mechanism and Information Supervision of Public Opinions in Internet Emergency, Procedia Computer Science, Vol. 17 (2013) No.5, p.973

[3] K.F Xie, S Zhao , CHEN G and W.J Cai: Research on Lifecycle Principle and Group Decision-making of Network Public Sentiment Emergency, Journal of Wuhan University of Technology, Social Sciences Edition, Vol. 23 (2010) No. 4, p.006.(In Chinese)

[4] H.L. Song, N.Q. Ju, B. Zhang and X.J. Pu: Formation, Evolution and Control of Network Public Opinion for Emergencies, Journal of Henan Institute of Engineering(Social Science Edition), Vol. 25 (2010) No.4, p. 12.( In Chinese)

[5] Y. ZHANG, P. Luuo, Q.L. Liu: A Study in Monitoring of Network Public Opinion Based on Life Cycle Theory, Information Science, Vol. 31 (2013) No. 11, p.006.(In Chinese)

[6] H.Q. Li, Y.X. Lan, X.N Hou and Q. Zhang: Study on the Theoretical Basis of the Network Public Opinion Management, Journal of Modern Information, Vol. 35 (2015) No.5, p. 25. (In Chinese) 
[7] G. Li and J.H. Chen: A Review of Network Public Opinion for Unexpected Emergency, Documentation, Information \& Knowledge, Vol. 158 (2014) No.2, p. 111.(In Chinese)

[8] S. Luthra, S. Mangla, L Xu and A. Diabat: Using AHP to evaluate barriers in adopting sustainable consumption and production initiatives in a supply chain, International Journal of Production Economics, Vol. 181 (2016) No. 11, p.342

[9] R. Liu, S.T. Bian and Q. Yu: Application of Evidence Theory in Quality Evaluation Based on Fuzzy Remark Remark Set, Journal of Wuhan University of Technology(Information \& Management Engineering), Vol. 35 (2013) No.3, p.448.(In Chinese)

[10]D.F Wu and H.C Huang: A Fuzzy-Grey MultiFactorial Evaluation on Technology Innovation Ability of the Emergency Management under The Background of Rapid Urbanization, Technology and Innovation Management, Vol. 36 (2015) No.4, p.388.(In Chinese) 